\title{
Synthesis of epitaxial monolayer Janus SPtSe
}

Roberto Sant ${ }^{1,2}$, Maxime Gay $\mathbb{D}^{3}$, Alain Marty $\mathbb{D}^{4}$, Simone Lisi ${ }^{1}$, Rania Harrabi ${ }^{5}$, Céline Vergnaud ${ }^{4}$, Minh Tuan Dau ${ }^{4}, \mathrm{Xiaorong} \mathrm{Weng}^{5}$, Johann Coraux (iD) ${ }^{1}$, Nicolas Gauthier ${ }^{3}$, Olivier Renault $\mathbb{D}^{3}$, Gilles Renaud ${ }^{5 凶}$ and Matthieu Jamet $\mathbb{I D}^{4 凶}$

Janus single-layer transition metal dichalcogenides, in which the two chalcogen layers have a different chemical nature, push chemical composition control beyond what is usually achievable with van der Waals heterostructures. Here, we report such a Janus compound, SPtSe, which is predicted to exhibit strong Rashba spin-orbit coupling. We synthetized it by conversion of a single-layer of $\mathrm{PtSe}_{2}$ on $\mathrm{Pt}(111)$ via sulfurization under $\mathrm{H}_{2} \mathrm{~S}$ atmosphere. Our in situ and operando structural analysis with grazing incidence synchrotron X-ray diffraction reveals the process by which the Janus alloy forms. The crystalline long-range order of the as-grown $\mathrm{PtSe}_{2}$ monolayer is first lost due to thermal annealing. A subsequent recrystallization in presence of a source of sulfur yields a highly ordered SPtSe alloy, which is isostructural to the pristine $\mathrm{PtSe}_{2}$. The chemical composition is resolved, layer-by-layer, using angleresolved X-ray photoelectron spectroscopy, demonstrating that Se-by-S substitution occurs selectively in the topmost chalcogen layer.

npj 2D Materials and Applications (2020)4:41; https://doi.org/10.1038/s41699-020-00175-z

\section{INTRODUCTION}

Most electronic properties of crystals are inherited from their symmetries. For monolayer transition metal dichalcogenides (TMDCs) in the $1 \mathrm{H}$ phase like $\mathrm{MX}_{2}(\mathrm{M}=\mathrm{Mo}, \mathrm{W}$ and $\mathrm{X}=\mathrm{S}, \mathrm{Se})$, the mirror symmetry with respect to the transition metal atoms plane leads to zero internal out-of-plane electric field and suppresses any Rashba spin-orbit coupling (SOC). The same happens for monolayer TMDCs in the $1 \mathrm{~T}$ phase like $\mathrm{PtSe}_{2}$ because of crystal inversion symmetry. In both phases, the application of a vertical electric field lowers the symmetry and can induce spin-splitting of the electronic bands due to the Rashba $\mathrm{SOC}^{1,2}$. The resulting spin texture is at the origin of unique spintronics phenomena ${ }^{3}$. An alternative route consists in breaking the out-of-plane mirror symmetry (in $1 \mathrm{H}$ phases) or the crystal inversion symmetry (in 1T phases) by substituting one of the two chalcogen layers with a layer of another chalcogen species. The resulting ternary compounds, so-called Janus after the biface Roman god, are predicted to exhibit strong Rashba SOC, possibly altered by mechanical stress and external electric fields ${ }^{4,5}$. The internal electric field in Janus TMDCs may be exploited also in piezoelectric devices $^{6}$ and $p-n$ junctions ${ }^{7}$. Anionic (chalcogen) substitution reactions have been used to tune the composition of TMDCs, with the main purpose of engineering the bandgap ${ }^{8-10}$. However, few reports addressed so far the selective substitution of the chalcogen atoms in a single layer (SL) to prepare ordered Janus compounds ${ }^{11,12}$. SL $1 \mathrm{~T} \mathrm{PtSe} \mathrm{P}_{2}$ is an indirect bandgap semiconductor $^{13}$ with a high spin-orbit interaction. It exhibits interesting magnetic properties, such as helical spin texture ${ }^{14}$, hidden spin polarization connected to spin-layer locking ${ }^{14}$, defect-induced magnetic ordering ${ }^{15}$, and magnetic anisotropy ${ }^{16}$. The first epitaxial synthesis of $\mathrm{SL} \mathrm{PtSe}{ }_{2}$ was performed by direct selenization of a Pt (111) crystal surface ${ }^{13}$. Since then, other growth approaches have been reported, including chemical vapor deposition ${ }^{17}$, molecular beam epitaxy ${ }^{18}$, plasma assisted selenization ${ }^{19}$, and thermally assisted conversion of predeposited metal layers ${ }^{20}$.
Here, we synthesized epitaxial SL PtSe $\mathrm{P}_{2}$ by selenization of a $\mathrm{Pt}$ (111) single crystal, and we developed a method for the conversion of the as-grown material into the Janus SPtSe by sulfurization of $\mathrm{PtSe}_{2}$ using $\mathrm{H}_{2} \mathrm{~S}$ gas. The whole process has been monitored in situ under ultrahigh vacuum (UHV) or under $\mathrm{H}_{2} \mathrm{~S}$ partial pressure of $10^{-4}$ mbar by synchrotron grazing incidence $\mathrm{X}$ ray diffraction (GIXRD). These experiments (i) confirmed that the coincidence lattice superstructure stemming from the mismatch between $\mathrm{PtSe}_{2}$ and $\mathrm{Pt}(111)$ is long-range ordered ${ }^{13}$; (ii) revealed a perfect epitaxial alignment of $\mathrm{PtSe}_{2}$ along the main $\mathrm{Pt}(111)$ crystallographic directions, but with sizeable lattice distortions in both the $\mathrm{PtSe}_{2}$ and the topmost $\mathrm{Pt}(111)$ layers, in contrast to what would occur if the epitaxy was of van der Waals (vdW) type; and (iii) proved the structural pinning of the two-dimensional (2D) layer to the substrate, the coincidence lattice remaining unaltered after the sulfurization of the pristine $\mathrm{PtSe}_{2}$. Finally and most importantly, we demonstrated that sulfurization under carefully controlled temperature conditions leads to a layer-selective substitution, and hence to an ordered Janus material, where $S$ atoms replace Se ones only in the top layer, as demonstrated by angle-resolved X-ray photoemission spectroscopy (AR-XPS). Longer exposure to $\mathrm{H}_{2} \mathrm{~S}$ at higher temperature further allows to form a platinum disulfide $\left(\mathrm{PtS}_{2}\right) \mathrm{SL}$ isostructural to the pristine $\mathrm{PtSe}_{2}$ one and pinned to the $\mathrm{Pt}(111)$ substrate by substituting all the Se atoms with $\mathrm{S}$ atoms.

\section{RESULTS AND DISCUSSION}

$\mathrm{PtSe}_{2}$ growth on $\mathrm{Pt}(111)$ and structural analysis

Before we address the formation and structure of the Janus alloy, we discuss about its parent compound, the pristine $\mathrm{SL} \mathrm{PtSe}{ }_{2}$. SL $\mathrm{PtSe}_{2}$ was grown in UHV by a two-step process based on the direct selenization of a Pt(111) crystal surface (see "Methods" for details), as described by Wang et al. ${ }^{13}$. Firstly, several $\mathrm{nm}$ of amorphous selenium were deposited on top of $\mathrm{Pt}(111)$ until the reflection high-energy electron diffraction (RHEED) pattern disappeared.

\footnotetext{
${ }^{1}$ Université Grenoble Alpes, CNRS, Institut Néel, 38042 Grenoble, France. ${ }^{2}$ ESRF, the European Synchrotron, 38043 Grenoble, France. ${ }^{3}$ Université Grenoble Alpes, CEA, LETI, 38054 Grenoble, France. ${ }^{4}$ Université Grenoble Alpes, CEA, CNRS, Grenoble INP, IRIG-Spintec, 38054 Grenoble, France. ${ }^{5}$ Université Grenoble Alpes, CEA, IRIG-MEM, 38054 Grenoble, France.

区email: gilles.renaud@cea.fr; matthieu.jamet@cea.fr
} 

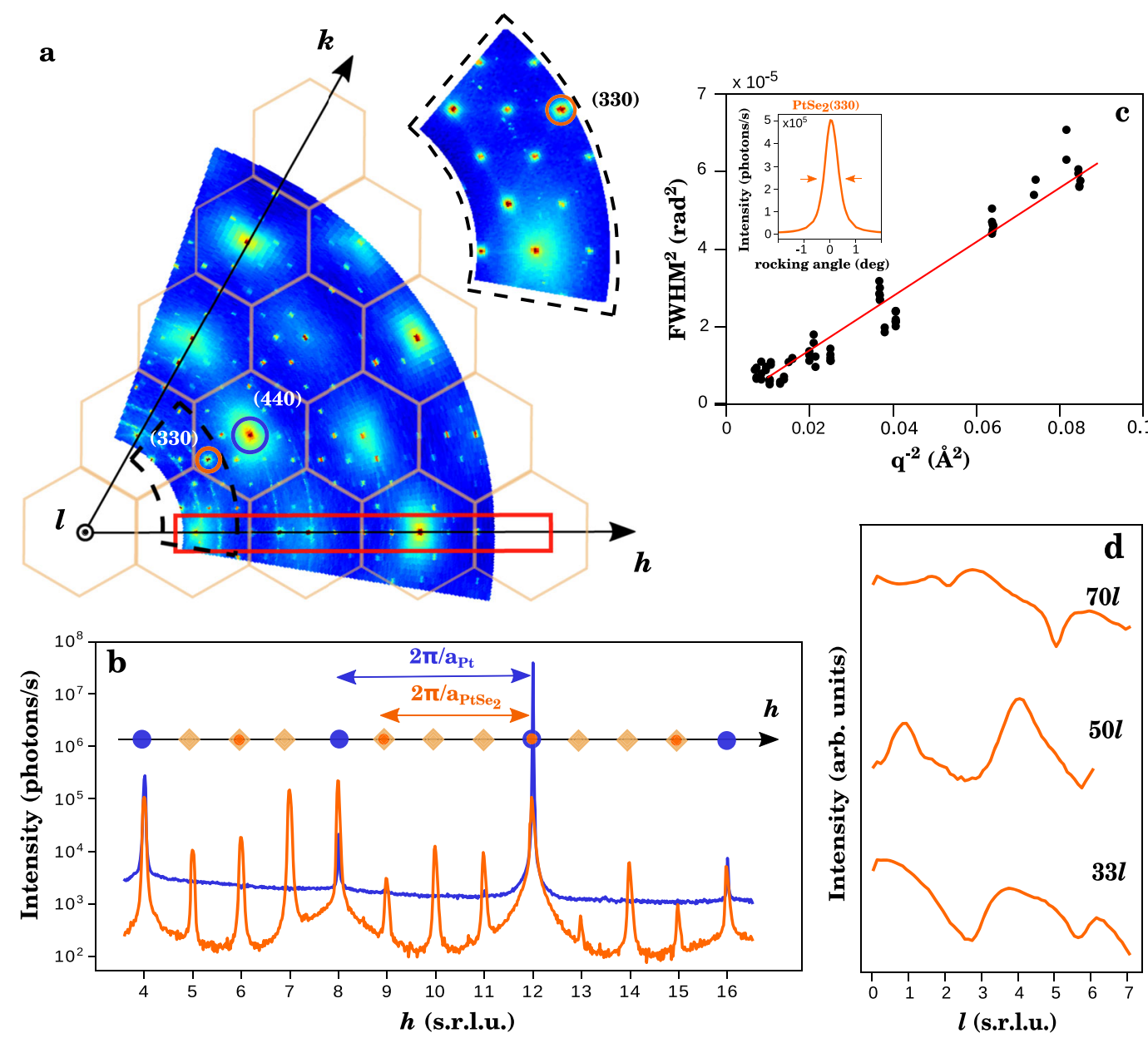

Fig. 1 GIXRD characterization of $\mathrm{PtSe}_{2} 2$ grown by selenization of $\mathrm{Pt}(111)$. a $\mathrm{A} 80^{\circ}$ sector map of the reciprocal space parallel to the surface at $I=0$, as acquired after the selenization; $(h, k, l)$ are surface reciprocal lattice units expressed in terms of the coincidence supercell; the orange hexagonal frames delimit $\mathrm{Pt}(111)$ Brillouin zones; orange and blue circles highlight two in-plane peaks belonging, respectively, to PtSe ${ }_{2}$ and the Pt substrate lattices. The rings of scattered intensity centered around the origin can be indexed as polycrystalline grains Pt reflections, very likely originated from damaged areas of the single crystal surface. The inset on the right shows a highly resolved diffraction pattern corresponding to the black-dashed-line-delimited sector in the extended map; the image has been acquired from a different sample without Pt polycrystalline grains. b Highly resolved in-plane radial scans measured along the high symmetry direction $h$ (highlighted in red in a) of the $\mathrm{Pt}$ (111) substrate before (blue) and after (orange) the selenization; the $h$ axis is indexed using supercell reciprocal lattice units (s.r.l.u.); blue and orange filled circles indicates $\mathrm{Pt}(111)$ and $\mathrm{PtSe}_{2}$ reflections, whereas the remaining peaks are associated to superstructure peaks by orange diamonds. c Plot and linear fit of the squared transversal width of the in-plane peaks measured by rocking scans as a function of the inverse squared modulus of the transfer vector q. The intercept of the fit with the vertical axis provides an estimation of the (negligible) overlayer mosaic spread. The slope of the linear fit allows deducing the average $\mathrm{PtSe}_{2}$ domain size. d Scans along the out-of-plane direction $I$ perpendicular to the sample surface at the positions (33/), (50/), and (70), relative to a PtSe ${ }_{2}$ Bragg peak and two superstructure peaks. The logarithm of the intensity is plotted.

Secondly, the sample was annealed from room temperature (RT) to $370^{\circ} \mathrm{C}$. During the annealing, the platinum atoms bond to selenium at the interface forming a $\mathrm{PtSe}_{2}$ monolayer, whereas the excess selenium desorbs from the surface.

Figure 1 a shows an $80^{\circ}$ in-plane sector of the reciprocal space mapped with GIXRD after the selenization. This map displays the successive Brillouin zones of $\mathrm{Pt}(111)$ (orange hexagons) having sharp $\mathrm{Pt}(111)$ peaks at their centers, with additional sharp peaks corresponding exactly to a $(4 \times 4)$ superstructure relative to the $\mathrm{Pt}$ (111) lattice. In Fig. 1b, two high-resolution linear scans measure the scattered intensity along the radial direction $h$ (highlighted by the red frame in Fig. 1a) before (blue) and after (orange) the selenization. The Pt peaks are pointed out by blue filled circles. The $\mathrm{PtSe}_{2}$ peaks, labeled with orange filled circles, are characterized by a periodic reciprocal lattice vector, which is three quarters the $\mathrm{Pt}(111)$ surface one, consistent with previous reports ${ }^{13}$. Additional peaks, marked by orange diamonds in between the $\mathrm{Pt}$ and $\mathrm{PtSe}_{2}$ peaks are perfectly aligned and equi-spaced. This diffraction pattern is the signature of a superlattice, which is the coincidence between $(3 \times 3) \mathrm{PtSe}_{2}$ and $(4 \times 4) \mathrm{Pt}(111)$ surface cells, and has a superperiodicity of $11.1 \AA$, in agreement with ref. ${ }^{13}$. The reciprocal space is indexed using this superstructure unit cell, so that the $\mathrm{Pt}(111)$ and $\mathrm{PtSe}_{2}$ Bragg peaks are at $h$ and $k$ surface reciprocal lattice units (s.r.l.u.) multiple of 4 and 3 respectively. We deduced a PtSe $e_{2}$ surface lattice constant of $(3.700 \pm 0.002) \AA$, slightly smaller (by $0.7 \%$ ) than the relaxed bulk value of $3.724 \AA^{21}$, and a very good epitaxial alignment between the $\mathrm{PtSe}_{2}$ monolayer and the Pt substrate. From the analysis of the width of $\mathrm{PtSe}_{2} \mathrm{Bragg}$ peaks in transverse scans (see Fig. 1c and Supplementary Note 1), we deduced a finite domain size of $240 \AA$ and a negligibe in-plane mosaicity. From the analysis of radial scans along the $(h 00)$ and (hho) crystallographic directions (see Supplementary Note 1), we obtained a very similar domain size of $220 \AA$ and an estimation of the relative inhomogeneous strain of $\Delta a / a \approx 3 \times 10^{-6}$. The global compressive strain of $0.7 \%$ and the perfect angular alignment are signatures of a strong interaction between $\mathrm{PtSe}_{2}$ and $\mathrm{Pt}(111)$. 
This hypothesis is supported by the remarkably strong intensity of the superstructure peaks, similar or exceeding those of $\mathrm{PtSe}_{2}$ peaks nearby (e.g., compare peaks at $h=7$ and $h=6$ ). It indicates sizeable distortion in the two surface lattices. In Fig. 1d (other rod scans are shown in Supplementary Note 2 and Supplementary Fig. $1)$, the intensity profiles recorded along the / direction perpendicularly to the surface for the superstructure peaks exhibit broad peaks and dips. The perpendicular coordinate $I$ is expressed in reciprocal lattice units of the Pt substrate unit cell with basal plane parallel to the $\mathrm{Pt}(111)$ surface, and of parameter $c=6.797 \AA$ equal to the thickness of three $\mathrm{Pt}(111)$ planes in the bulk. Notably, most superstructure peaks (except those of $\mathrm{PtSe}_{2}$ with $h$ and $k$ multiple of 3 ), are located close to integer values of $I$ (see e.g., (50/) rod in Fig. 1d), which are Bragg conditions for Pt(111). As already discussed in the past for other surface systems ${ }^{22}$, this indicates that the interlayer periodicity in the substrate contributes substantially to the superstructure rods shapes and that lattice distortions are present not only in the $\mathrm{PtSe}_{2} \mathrm{SL}$ but also in the few topmost Pt layers ${ }^{23}$. Moreover, from the superstructure rods, we can confirm the monolayer character of $\mathrm{PtSe}_{2}$ (see Supplementary Note 3).

Obviously, our $\mathrm{PtSe}_{2} / \mathrm{Pt}(111)$ system cannot be described using the vdW epitaxy picture, where the $2 \mathrm{D}$ overlayer grows almost strain-free due to very weak interaction with its substrate. It rather implies a strong coupling between $\mathrm{PtSe}_{2}$ and the $\mathrm{Pt}(111)$ surface. Such a strong interaction has important consequences in the structural constraints inherited by the material upon sulfurization. In particular, as shown below, it helps keeping the integrity of the $\mathrm{PtSe}_{2}$ crystal during annealing and after the sulfurization process.

\section{Stability of the as-grown $\mathrm{PtSe}_{2}$ under annealing}

In situ measurements during annealing in UHV have been performed to determine the temperature window for which the epitaxial $\mathrm{PtSe}_{2} \mathrm{SL}$ is stable. Figure 2a displays ( $\left.h 00\right)$ radial scans of the scattered X-ray intensity containing the second-order $\mathrm{PtSe}_{2}$ reflection (at $h=6$ ), two superstructure peaks (at $h=5$ and $h=7$ ) and two Pt(111) peaks (at $h=4$ and $h=8$ ). The superstructure and $\mathrm{PtSe}_{2}$ peak intensities progressively decrease with time when the annealing temperature exceeds the growth temperature of $370^{\circ} \mathrm{C}$, until they vanish completely (spectra labeled a-d in Fig. 2a). We deduce that above the growth temperature the annealing not only lifts the superstructure order (i.e., the $\mathrm{PtSe}_{2}$ and $\mathrm{Pt}$ lattice constants are not in a 3:4 ratio anymore), but also degrades the $\mathrm{PtSe}_{2}$ structure. Surprisingly, cooling down to $300^{\circ} \mathrm{C}$ or lower leads to the recrystallization of the $\mathrm{PtSe}_{2}$ layer and restores the original superstructure, as evident in the diffraction patterns (compare spectra labeled $d-g$ and RT in Fig. 2a). The most plausible scenario accounting for these observations is that the Se-Pt bonds break at annealing temperatures above $370^{\circ} \mathrm{C}$, and yield a disordered, possibly highly mobile, phase of selenium atoms onto the substrate surface, as suggested by the background increase in the scans taken at intermediate temperatures (not shown) compared to the RT scan. The degradation of $\mathrm{PtSe}_{2}$ and the concomitant release of $\mathrm{Se}$ atoms appear to be progressive, occurring over a time range of several tens of minutes. The domain size is apparently not affected since the width of the inplane peaks is unchanged. This suggests point defects, for instance chalcogen vacancies, as the dominant type of disorder in the system. Cooling at RT restores the pristine structure, i.e., free Se atoms are consumed for the recrystallization of $\mathrm{PtSe}_{2}$, e.g., by filling vacancies. This also implies that the Se desorption rate is marginal at the explored temperatures.

\section{Sulfurization of $\mathrm{PtSe}_{2}$ in $\mathrm{H}_{2} \mathrm{~S}$ atmosphere}

So far, we have discovered that a reversible path can be followed from dissolution to recrystallization of $\mathrm{PtSe}_{2}$ upon cycles of annealing and cooling. At this point, we wondered whether, after degradation of the $\mathrm{PtSe}_{2}$, one could follow a new cooling path in the presence of a sulfur precursor, leading to an ordered chalcogen alloyed SL TMDC, via substitution of some Se atoms by $\mathrm{S}$ ones, thanks to the higher stability of Pt-S bonds compared to Pt-Se ones. To test this proposal, we supplied sulfur in the form of gaseous $\mathrm{H}_{2} \mathrm{~S}$, which is known to decompose on noble metal surfaces.

We prepared a first sample called SPtSe by pre-annealing it in UHV a few tens of degrees above the growth temperature (between $370^{\circ} \mathrm{C}$ and $400^{\circ} \mathrm{C}$ ), in order to create Se vacancies in the topmost chalcogen layer, which will be eventually filled with $S$ atoms. Then, a $10^{-4}$ mbar $\mathrm{H}_{2} \mathrm{~S}$ partial pressure was introduced in the UHV chamber and maintained for $5 \mathrm{~h}$ at a slightly lower temperature of $350^{\circ} \mathrm{C}$. We monitored the sulfurization process by GIXRD to track possible structural changes due to the chalcogen substitution in the $\mathrm{PtSe}_{2}$ lattice, e.g., strain, loss of the superstructure order, surface disorder, new phases with different lattice parameters, or additional/suppressed peaks corresponding to different crystal symmetries. In Fig. $2 b$, the radial scans of asgrown $\mathrm{PtSe}_{2}$ and sulfurized $\mathrm{PtSe}_{2}$ are compared. After $\mathrm{H}_{2} \mathrm{~S}$ treatment, the intensity loss $(-58 \%)$ of the $(600) \mathrm{PtSe}_{2} \mathrm{Bragg}$ peak and the broader tail in the low $h$ values of the Pt(400) crystal truncation rod (CTR) peak suggest an increased surface disorder ${ }^{24}$. Except for that, the spectra are remarkably similar to those of pristine $\mathrm{PtSe}_{2} / \mathrm{Pt}(111)$, the $(3 \times 3)$-on- $(4 \times 4)$ coincidence being exactly maintained. The chemical composition of this SPtSe sample was probed by XPS. The Se $3 s / S 2 s$, Se $3 p / S 2 p$, and Se $3 d$ core-level $(\mathrm{CL})$ spectra are shown in Fig. 3, and compared to those of a reference sample of pristine $\mathrm{PtSe}_{2}$ prepared by selenization. We clearly see almost equal contributions from $\mathrm{S}$ and Se demonstrating the partial sulfurization of this sample.

In order to achieve full sulfurization, we prepared a second sample called $\mathrm{PtS}_{2}$ by exposing $\mathrm{PtSe}_{2}$ to $\mathrm{H}_{2} \mathrm{~S}$ partial pressure for a longer time ( $9 \mathrm{~h}$ in total) after a pre-annealing in UHV at a higher temperature (up to $460^{\circ} \mathrm{C}$ ). The XPS spectra in Fig. 3 show a clear predominant sulfur content with little traces of selenium. Noteworthy, the very intense Se Auger lines at 174 and $178.5 \mathrm{eV}$ visible for $\mathrm{PtSe}_{2}$ are particularly weak for this sample. The sulfurization was also monitored in operando by GIXRD. Similarly to Fig. 2b, c shows two radial scans along the $h$ direction taken before (black) and after (red) the sulfurization process. Here, the peak intensity at $h=6$ (previously $\mathrm{PtSe}_{2}$ reflection) is severely damped to about one tenth of the pristine intensity and becomes asymmetric, the intensity at the Pt CTR tails (on the low- $h$ sides of the $h=4$ and $h=8$ peaks) is higher and all the peaks are wider, all suggesting disorder in the surface layer. The $\mathrm{SL} \mathrm{PtSe} \mathrm{P}_{2}$ overlayer is very likely degraded due to the higher temperature used and to the longer exposure to $\mathrm{H}_{2} \mathrm{~S}$. However, remarkably, a residual ordered superstructure is still preserved, which can be hardly associated to any pure $\mathrm{PtSe}_{2}$ or SPtSe alloy since XPS rules out the presence of Se. No other diffraction signals were found which could be attributed to a relaxed $\mathrm{PtS}_{2}$ or other phases. It is worth noting that for $\mathrm{PtS}_{2}$ to be in a 4:3 ratio with $\mathrm{Pt}(111)$, conditions required by the superstructure, it should stretch its lattice parameter by as much as $4.6 \%$. The expected Bragg peak position in Fig. $2 \mathrm{c}$ is indicated by a black arrow at $h=6.28$. Therein, the only new detectable signatures are some peak shoulders, that we ascribe to either sulfide phases of slightly shorter lattice parameter than $\mathrm{PtSe}_{2}$ coexisting with the superstructure or the presence of defects yielding a diffuse scattering contribution. A plausible interpretation of our observations is that the $\mathrm{H}_{2} \mathrm{~S}$ supply at elevated temperature has allowed for a replacement of all Se atoms by $\mathrm{S}$ atoms. Similarly, no substantial modifications of the pristine diffraction pattern were observed in the small map of Fig. $2 \mathrm{~d}$. The only significant changes are the clear elongation of most of the superstructure peaks toward the (440) $\mathrm{PtSe}_{2}$ main reflection. These elongations might arise from specific defects that would need a very extensive investigation, which is beyond the scope of 


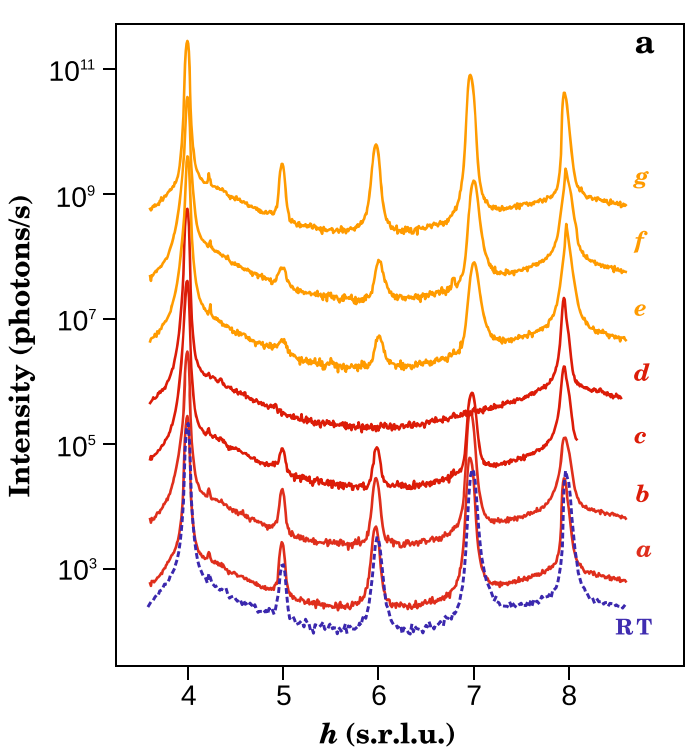

d

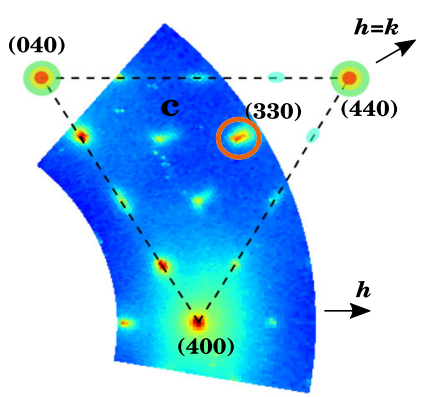

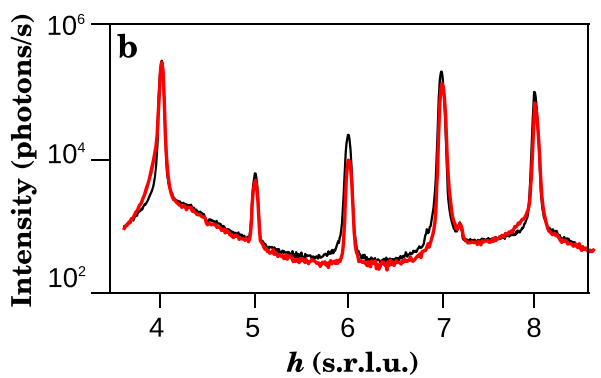

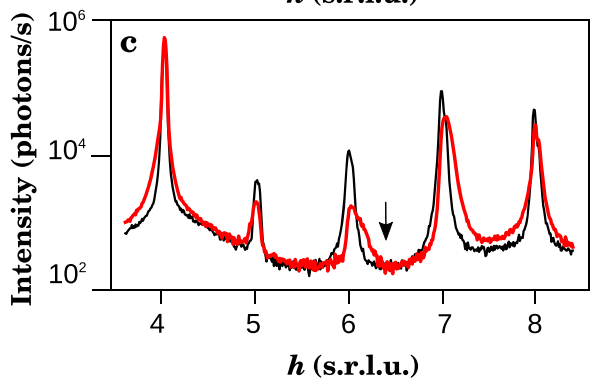

$\mathbf{f}$

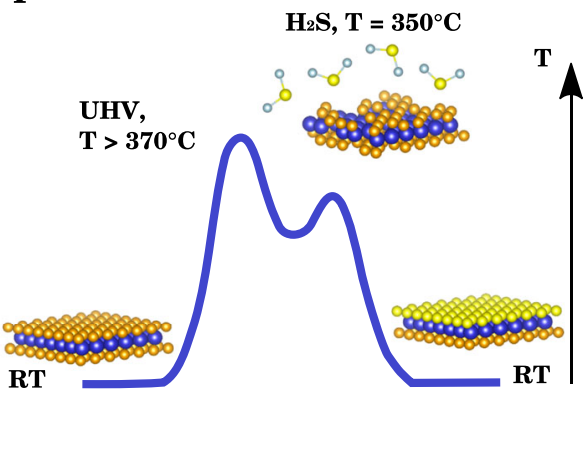

Fig. 2 Annealing and sulfurization of $\mathrm{PtSe}_{2}$ in $\mathbf{H}_{2} \mathbf{S}$ atmosphere. a Evolution of the radial scan measured along the $h$ axis between $h=3.8$ and $h=8.4$ after annealing at a temperature higher than $370^{\circ} \mathrm{C}$ (from a to d) and subsequent stabilization at $300^{\circ} \mathrm{C}$ (from d to g); the dashed blue profile at the bottom is measured at room temperature before the annealing and is superimposed (with no offset) to curve a to highlight the increment of the background. b Same radial scan along the $h$ axis, before (black) and after (red) partial sulfurization leading to a Janus structure. c Same radial scan along the $h$ axis, before (black) and after (red) complete sulfurization of the PtSe ${ }_{2}$ layer; the black arrow indicates the expected position for a freestanding (unstrained) $\mathrm{PtS}_{2}$ Bragg reflection. $\mathbf{d}$ In-plane reciprocal space map equivalent to that shown in the inset of Fig. 1a corresponding to a fully sulfurized $\mathrm{PtS}_{2}$ : the peak positions remain unaltered with respect to the as-grown PtSe $\mathrm{e}_{2}$ case, although chalcogen substitution is demonstrated by photoemission spectroscopy. Remarkably, most of the peaks display diffuse scattering tails pointing toward the $\mathrm{Pt}(440)$ reflection. e Scans along the out-of-plane direction / perpendicular to the sample surface at the positions (33/), (50/), and (70/), relative to a PtSe $e_{2}$ Bragg peak and two superstructure peaks, before (orange) and after (green) the complete sulfurization of the $\mathrm{PtSe}_{2}$ monolayer. $\mathbf{f}$ Pictorial scheme of the sulfurization process for the transformation of $\mathrm{PtSe}_{2}$ into a Janus material: PtSe $\mathrm{P}_{2}$ is first annealed in vacuum at temperatures slightly higher than the growth temperature $\left(370^{\circ} \mathrm{C}\right.$ ); subsequently, it is exposed to $\mathrm{H}_{2} \mathrm{~S}$ gas at $350^{\circ} \mathrm{C}$ where the defects and vacancies created during the first step become ideal sites for $\mathrm{H}_{2} \mathrm{~S}$ adsorption and Se-by-S substitution. The process ends up with the conversion of the pristine $\mathrm{PtSe}_{2}$ into a Janus SPtSe material.

the present study. Some scattering rods produced by the superstructure were also measured on both sulfurized samples (Fig. 2e). Except for their different intensity and faster decrease with $I$, they show qualitatively exactly the same features as the original $\mathrm{PtSe} \mathrm{S}_{2}$ ones. This confirms that, even for the fully sulfurized sample, the original $\mathrm{PtSe}_{2}$ structure is preserved.

To summarize, the in-plane diffraction pattern and the out-ofplane rods of the as-grown $\mathrm{PtSe}_{2}$ remain almost unchanged upon the sulfurization procedures, while $S$ atoms substitute some or all of Se atoms (Fig. 2b-e) as shown with XPS, yielding a SPtSe alloy and pure $\mathrm{PtS}_{2}$ isostructural to the pristine $\mathrm{PtSe}_{2}$ (although with some disorder that we attribute to the creation of defects). Hence, all along the recrystallization, the memory of the initial $\mathrm{PtSe}_{2}$ pinning on its substrate is maintained, and the substitution of the chalcogen atoms occurs without significant modification of their lateral position with respect to the substrate atoms. Both the strong interaction between $\mathrm{Pt}$ and $\mathrm{PtSe}_{2}$ and the fortunate 4-3 coincidence of the in-plane lattice parameters are believed to lead to this pinning. This conclusion is a further evidence that the epitaxy in this system, rather than being of vdW type, is governed by more covalent interface interactions leading to distortions in both the overlayer and the substrate. These interactions probably explain why the $\mathrm{PtSe}_{2}$ crystal retains its crystal integrity upon sulfurization. In the following, we demonstrate that the sulfurization process of the SPtSe sample follows a scenario similar to that depicted in Fig. $2 f$.

\section{Evidence for a Janus alloy}

To probe the chemical composition of the SPtSe layer and estimate the vertical distribution of $\mathrm{S}$ and Se atoms, we performed AR-XPS measurements, as illustrated in Fig. 4a. Prior to the measurements, the samples were systematically annealed in vacuum, in order to remove any trace of $\mathrm{H}_{2} \mathrm{~S}$ that might be 

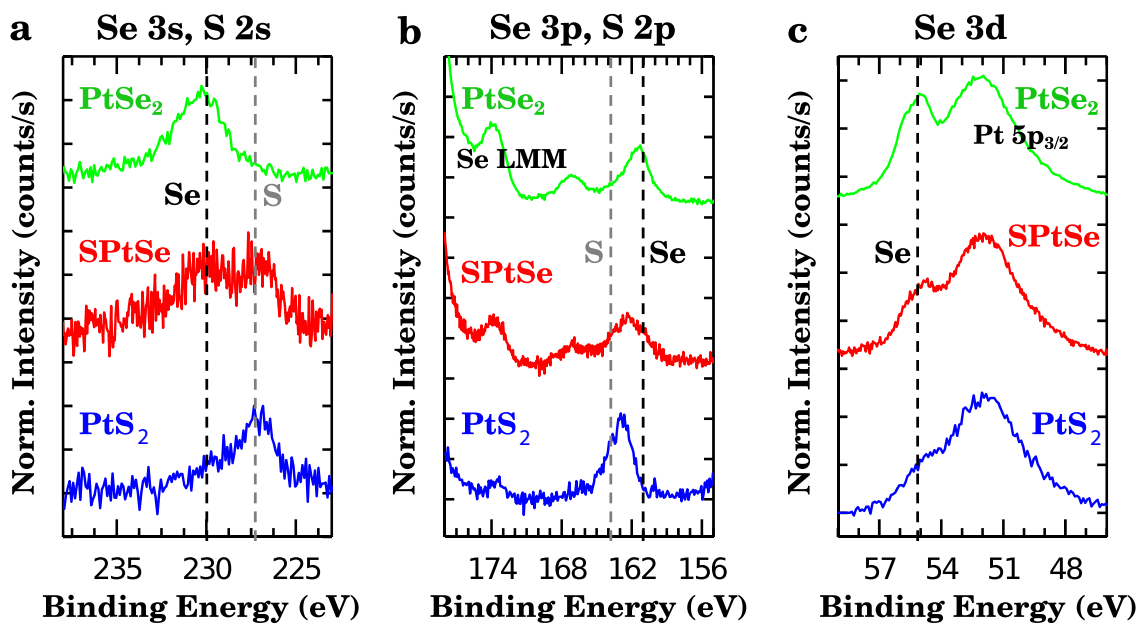

Fig. 3 XPS measurements on PtSe ${ }_{2}$, SPtSe, and sulfide samples. Se and S CLs of the different samples studied in this work, i.e., a pristine non-sulfurized $\mathrm{PtSe}_{2}$ (green), the Janus SPtSe (red), and a fully substituted $\mathrm{PtS}_{2}$ (blue). The take-off angle is $45^{\circ}$. From a to c different binding energy ranges are shown: a Se $3 s$ and $\mathrm{S} 2 \mathrm{~s}$, b Se $3 p$ and $\mathrm{S} 2 d$, and $\mathbf{c}$ Se $3 d$. All the samples were first annealed in vacuum prior to the measurements.

adsorbed at the surface after growth. In AR-XPS, the photoemission intensity varies with the depth of excitation of the photoelectrons, which is proportional to $\sin \theta, \theta$ being the takeoff angle between the surface and the spectrometer. The lower the take-off angle is, the smaller the probed depth and the higher the surface sensitivity are. Exploiting this effect allows determining whether sulfur substitutes selenium in the TMDC and whether the substitution occurs in the top or in the bottom layer, or in both. Figure $4 \mathrm{~b}-\mathrm{d}$ shows the Se $3 p$ and $S 2 p \mathrm{CL}$ spectra after subtraction of a Shirley background, fitted by two spin-orbit doublets for three different take-off angles $\theta=45^{\circ}, 25^{\circ}$, and $10^{\circ}$. The spin-orbit splitting of the Se $3 p$ and S $2 p$ CLs are fixed at 5.75 and $1.18 \mathrm{eV}$, respectively ${ }^{25,26}$. We find that the $S$ contribution increases when $\theta$ decreases, while the opposite trend is observed for Se. The ratio between the $S$ and Se contributions to the spectra decreases as a function of the probed depth, from 1.54 at $\theta=10^{\circ}$ (short depth) to 1.15 at $\theta=45^{\circ}$ (long depth), as shown in Fig. 4e. This result clearly shows that the $\mathrm{H}_{2} \mathrm{~S}$ treatment induces a Se-by-S substitution in the $\mathrm{PtSe}_{2}$ lattice preferentially in the top layer. It suggests also that the kinetic barriers to the substitution differ for the two chalcogen layers.

We then performed a quantitative analysis of the Se and $\mathrm{S} \mathrm{CL}$ intensities derived from the peak fitting of Fig. $4 b-d$ and from the spectra of Fig. 3 to determine the chalcogen vertical distribution in SPtSe by AR-XPS. This analysis faces two main challenges. Firstly, elastic scattering along the path taken by the photoelectrons toward the detector ${ }^{27}$ needs to be taken into account in a rigorous way. This effect is most prominent at grazing take-off angles as used here, and results in significant changes in the measured intensities emitted from buried layers. The correction procedure for elastic scattering is complex because it varies with the geometry of the experiment and the details of the sample composition. Secondly, when a sample consists of chemically different layers, the exact element-specific photoelectron inelastic mean free path (IMFP) must be taken into account for each of these layers.

For this work, we used the QUASES-AR-XPS software developped by Tougaard et al. (http://quases.com/products/quasesarxps/). In this approach, elastic scattering is accounted for by a relatively simple analytical expression of the depth-distribution function, which describes the measured angular-dependent intensity ${ }^{28,29}$. To quantitatively determine the Se and S concentrations in each of the chalcogen layers, we have considered as physical constraint and input the thickness of the SPtSe overlayer, and let the concentrations be derived from the angle-dependent $\mathrm{CL}$ intensity curve. The SPtSe thickness was calculated as follows: we used the attenuation law of the Pt substrate signal, considering the Pt $4 f \mathrm{CL}$ at $\approx 71.5 \mathrm{eV}$ before and after the formation of SPtSe. We found a thickness of $3.7 \AA$ (the details can be found in Supplementary Note 4). This value is close to the $\mathrm{PtSe}_{2} \mathrm{SL}$ thickness $\left(2.53 \AA\right.$ in ref. $\left.{ }^{13}\right)$ and confirms the monolayer character of SPtSe, consistent with the analysis of diffraction data (Supplementary Note 3). The other important inputs are related to the IMFPs and the asymmetry parameter which takes into account the geometry of the measurement at each take-off angle. The IMFP is element-specific and also depends on the photoelectron kinetic energy; it is determined from the Tanuma-Powell-Penn formula using the QUASES-IMFP routine. The asymmetry parameter is derived from ref. ${ }^{30}$, and accounts for the experiment geometry through the electron orbital symmetry, and the angle between X-rays and the sample surface. All the input parameters are summarized in Table 1. We eventually determined the Se concentration in the bottom chalcogen layer from the Se $3 d$ angle-dependent intensities, after fitting the Se $3 d$ Pt $5 p_{3 / 2} C L$ spectra corrected for elastic scattering. This calculation showed that the Se signal originates at $98.5 \%$ from the bottom chalcogen layer, with only negligible contributions both from the Pt layer and the topmost chalcogen one. We next considered the angle-dependent intensities of both Se $3 p$ and $S 2 p C L$ spectra of Fig. $4 b-d$, and found equal Se and $S$ total concentrations in the sample. We thus conclude that, within the estimated $15-20 \%$ uncertainty, the selenium is localized exclusively in the bottom layer, whereas all the Se atoms of the first layer have been substituted by $\mathrm{S}$ atoms. This result establishes that the sulfurization process leads to a highly ordered ternary compound in a Janus configuration isostructural to the pristine $\mathrm{PtSe}_{2}$ monolayer: in the conditions we used, thermal energy is just enough for $S$ atoms to selectively substitute the first Se layer, as schematically depicted in Fig. 2f. We also performed ex situ AR-XPS on the PtS sample. Those measurements demonstrated that a quasicomplete substitution of selenium by sulfur has occurred in this case. A very low Se concentration (3.5\%) is estimated at $45^{\circ}$ takeoff angle for the Se $3 p \mathrm{CL}$ (for more grazing angles the estimate comes with a prevailing uncertainty due to the lower signal-tonoise ratio). In these harsher sulfurization conditions, thermal energy seems high enough, and the time of the process sufficiently long, for $\mathrm{S}$ atoms to diffuse through the $\mathrm{PtSe}_{2}$, in order to reach and occupy selenium vacancies in the bottom Se 
a
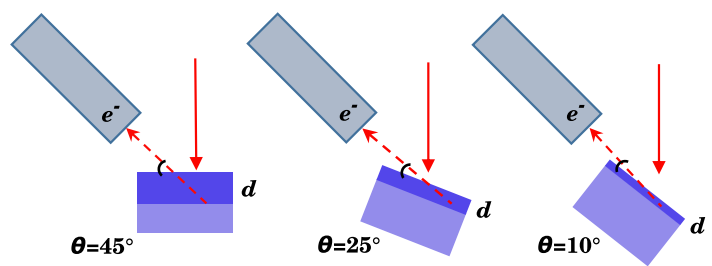

b

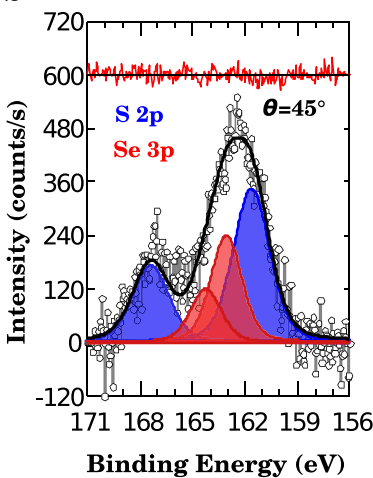

c

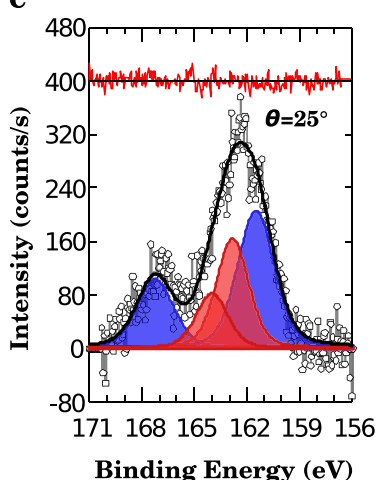

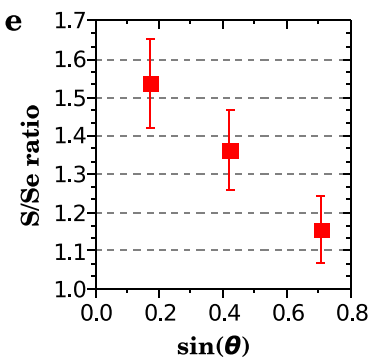

d

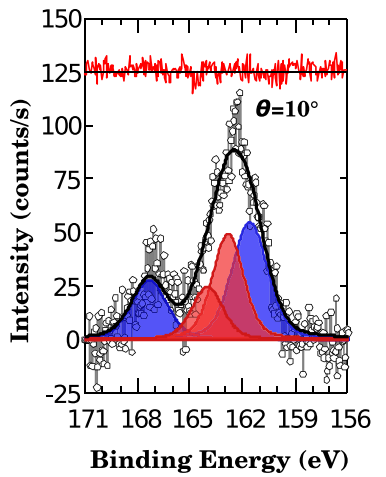

Fig. 4 Angle-resolved XPS core-level spectra of Janus SPtSe. a Sketch illustrating the AR-XPS technique and the relationship between the probing depth $d$ and the take-off angle $\theta\left(d=\lambda \sin (\theta)\right.$, where $\lambda$ is the attenuation length taking into account elastic scattering $\left.{ }^{29}\right)$. The probing depths are $11.5,28.2$, and $47.15 \AA$ for $10^{\circ}, 25^{\circ}$, and $45^{\circ}$ take-off angles, respectively. b-d X-ray photoelectron spectra of Se $3 p$ and $\mathrm{S} 2 p$ core levels, taken at $45^{\circ}, 25^{\circ}$, and $10^{\circ}$ take-off angle $\theta$ on the sulfurized sample. The red line above the spectra corresponds to the fitting residual. The spectra have been normalized to the intensity at the S $2 p$ core level. e S/Se concentration ratio as a function of the take-off angle $\theta$.

\begin{tabular}{|lcccc|}
\hline \multicolumn{5}{l}{ Table 1. Input parameters for the quantitative XPS analysis. } \\
\hline Photoelectron/layer & Pt 5p & Se 3p & S 2p & Se 3d \\
\hline Kinetic energy $(\mathrm{eV})$ & 1450 & 1300 & 1300 & 1450 \\
IMFP ( $\AA$ ) & 15.7 & 26.2 & 29.5 & 28.5 \\
Asymmetry parameter & 1.75 & 1.30 & 1.16 & 1.08 \\
Intensity at $10^{\circ}$ & 626.2 & 240.3 & 188.6 & 147.8 \\
Intensity at $25^{\circ}$ & 2702.6 & 924.9 & 648.9 & 541.1 \\
Intensity at $45^{\circ}$ & 6076.1 & 1561.5 & 813.0 & 850.1 \\
\hline
\end{tabular}

Kinetic energy, IMFP, asymmetry parameter, and intensity values at three take-off angles for the quantitative analysis of the AR-XPS intensities.

layer. This entails overcoming the kinetic barriers that allow selective substitution of the first chalcogen layer to achieve the complete substitution of Se atoms by $\mathrm{S}$ atoms, and the formation of a $\mathrm{PtS} \mathrm{S}_{2}$ monolayer isostructural to the pristine $\mathrm{PtSe}_{2}$ one.

In conclusion, we achieved the transformation of a $\mathrm{PtSe}_{2} \mathrm{SL}$ into an ordered SPtSe Janus SL. We found that epitaxial PtSe $e_{2}$ is strongly bonded to its $\mathrm{Pt}(111)$ substrate. This situation is at variance with vdW epitaxy, since here the $\mathrm{PtSe}_{2}$ layer is strained and highly oriented. $\mathrm{PtSe}_{2}$ was next converted into an epitaxial Janus SPtSe TMDC by exposure to $\mathrm{H}_{2} \mathrm{~S}$ at a temperature at which its structure is strongly altered. While cooling without $\mathrm{H}_{2} \mathrm{~S}$ leads to a recrystallization toward the initial $\mathrm{PtSe}_{2}$, the presence of the sulfur molecular precursor for well chosen temperature and exposure time allowed the transformation of the top Pt-Se bonds into Pt-S ones. The differing kinetic barriers for the Se-by-S substitution process in the top and bottom chalcogen layers allowed recrystallization in a Janus configuration. This highly ordered 2D TMDC, which is not found in nature, exhibits crystal inversion symmetry breaking. This is expected to translate into new physical properties, e.g., a spontaneous electric polarization and a piezoelectric character, as well as strong Rashba SOC.

\section{METHODS}

Samples preparation

$\mathrm{PtSe}_{2}$ was prepared in two different UHV chambers hosted in Grenoble (France). First, $\mathrm{Pt}(111)$ single crystal surfaces were prepared in the INS2 UHV CVD-MBE-diffraction chamber at the CRG/IF-BM32 beamline ${ }^{31}$ (www. esrf.eu/UsersAndScience/Experiments/CRG/BM32) at the European Synchrotron radiation Facility (ESRF, www.esrf.eu). Subsequently, selenium was deposited in a second MBE chamber located at the CEA Grenoble. Base pressures in the two systems are in the low $10^{-10} \mathrm{mbar}$ range. The selenization process for the formation of $\mathrm{PtSe}_{2}$ has been followed by in situ X-ray diffraction in the same INS2 UHV environment where the $\mathrm{Pt}(111)$ crystals were prepared. Samples were kept under UHV conditions $\left(10^{-8}\right.$ mbar) during the transfers between the two chambers by means of a UHV home-made suitcase compatible with the two UHV setups. Finally, XPS measurements were performed in a PHI 5000 VersaProbe II photoelectron spectrometer at the Nanocharacterization Platform (PFNC) of the Minatec Campus in Grenoble (France). The preparation of the (111) surface of the single crystals (MaTech) consists of several cycles of $\mathrm{Ar}^{+}$ion bombardment (initially at $1.0 \mathrm{kV}$ and then at $0.8 \mathrm{kV}$ ) at $3 \times 10^{-6} \mathrm{mbar}$ Ar partial pressure followed by annealing in UHV at $900^{\circ} \mathrm{C}$, until the surface shows a sharp and unreconstructed RHEED pattern. Amorphous selenium was deposited on the bare $\mathrm{Pt}(111)$ crystal surfaces at RT under a $10^{-6} \mathrm{mbar}$ partial pressure of Se for $2 \mathrm{~min}$. This corresponds to a several $\mathrm{nm}$ thick amorphous Se film. The selenization is achieved by annealing the selenium covered sample in UHV up to the optimized temperature of $370^{\circ} \mathrm{C}$ inside the INS2 chamber. Temperatures were measured with pyrometers. The formation and optimization of the $\mathrm{PtSe}_{2}$ structure and of the sulfurized structures were monitored in situ by GIXRD.

\section{INS2 diffractomer}

The selenization process and final PtSe $\mathrm{P}_{2}$ structure were studied at INS2 by GIXRD. In INS2, the $z$-axis diffractometer is coupled with a bending magnet $\mathrm{X}$-ray source and the growth chamber, allowing structural characterization and growth at the same time. The beam energy was set at $19.8 \mathrm{keV}$ and the incident angle at $0.18^{\circ}$, slightly below the critical angle for total reflection. The beam size on the sample is $25(\mathrm{H}) \times 300(\mathrm{~V}) \mu \mathrm{m}^{2}$, whereas the vertical divergence is as low as $0.13 \mathrm{mrad}$. The studied samples are $8 \mathrm{~mm}$ in diameter and are vertically mounted. The diffracted intensity is acquired with a MaxiPix 2D hybrid pixel detector $\left(1296 \times 256\right.$ pixels, $55 \times 55 \mu \mathrm{m}^{2}$ 
pixel size) developed at the ESRF and located at $0.7 \mathrm{~m}$ from the diffractometer homocenter.

\section{GIXRD data acquisition and integration}

Measurements of the in-plane diffracted intensity in the reciprocal space plane have been performed by means of rocking scans centered on the $\mathrm{PtSe}_{2}$ /superstructure peaks ${ }^{32}$. The analysis (2D data plotting, integration of the collected intensities) has been performed using PyRoD (software specialized in the treatment of GIXRD data taken with a 2D detector, under development at ESRF-BM32).

\section{Angle-resolved XPS}

The data were measured with a PHI 5000 VersaProbe II photoelectron spectrometer at the Nanocharacterization Platform (PFNC) of the Minatec Campus in Grenoble (France). We transferred the samples between the INS2 UHV growth chamber and the XPS chamber under inert nitrogen atmosphere to limit the contamination from ambient air. The samples were inserted in ultrahigh vacuum and excited by a $200-\mu \mathrm{m}$ diameter monochromatic Al-Ka $(h v=1486.6 \mathrm{eV}) \mathrm{X}$-ray light source. The source analyzer angle is $45^{\circ}$ and remains unchanged during the AR-XPS data acquisition, where the photoelectron take-off angle (sample-analyzer angle) varies. The spectral intensity also decreases drastically in the same time, imposing a higher acquisition time. The overall energy resolution (taking into account both the spectrometer and X-ray bandwidths) is $0.6 \mathrm{eV}$ for $C L$ spectra and we set the $C 1 s$ peak from the adventitious carbon to $284.8 \mathrm{eV}$ for calibration of the binding energy scale. Data correction and peak deconvolution were performed with the CasaXPS software, and we used the sensitivity factors provided by the instrument to perform the relative quantification of the elements.

\section{DATA AVAILABILITY}

The data that support the findings of this study are available from the corresponding author (G.R. and M.J.) upon reasonable request.

Received: 7 June 2020; Accepted: 20 October 2020; Published online: 20 November 2020

\section{REFERENCES}

1. Riley, J. M. et al. Negative electronic compressibility and tunable spin splitting in WSe ${ }_{2}$. Nat. Nanotechnol. 10, 1043 (2015).

2. Yuan, H. et al. Zeeman-type spin splitting controlled by an electric field. Nat. Phys. 9, 563 (2013).

3. Edelstein, V. M. Spin polarization of conduction electrons induced by electric current in two-dimensional asymmetric electron systems. Solid State Commun. 73, 233 (1990)

4. Cheng, Y. C., Zhu, Z. Y., Tahir, M. \& Schwingenschlögl, U. Spin-orbit-induced spin splittings in polar transition metal dichalcogenide monolayers. Europhys. Lett. 102, 57001 (2013).

5. Hu, T. et al. Intrinsic and anisotropic Rashba spin splitting in Janus transitionmetal dichalcogenide monolayers. Phys. Rev. B 97, 235404 (2018).

6. Dong, L., Lou, J. \& Shenoy, V. B. Large in-plane and vertical piezoelectricity in Janus transition metal dichalchogenides. ACS Nano 11, 8242 (2017).

7. Palsgaard, M., Gunst, T., Markussen, T., Thygesen, K. S. \& Brandbyge, M. Stacked Janus device concepts: abrupt pn-junctions and cross-plane channels. Nano Lett. 18, 7275 (2018).

8. $\mathrm{Ma}, \mathrm{Q}$. et al. Postgrowth tuning of the bandgap of single-layer molybdenum disulfide films by sulfur/selenium exchange. ACS Nano 8, 4672 (2014).

9. Feng, Q. et al. Growth of $\operatorname{MoS}_{2(1-x)} \mathrm{Se}_{2 x}(x=0.41-1.00)$ monolayer alloys with controlled morphology by physical vapor deposition. ACS Nano 9, 7450 (2015).

10. Taghinejad, $H$. et al. Defect-mediated alloying of monolayer transition-metal dichalcogenides. ACS Nano 12, 12795 (2018)

11. Lu, A.-Y. et al. Janus monolayers of transition metal dichalcogenides. Nat. Nanotechnol. 12, 744 (2017).

12. Zhang, J. et al. Janus monolayer transition-metal dichalcogenides. ACS Nano 11, 8192 (2017).

13. Wang, Y. et al. Monolayer $\mathrm{PtSe}_{2}$, a new semiconducting transition-metal-dichalcogenide, epitaxially grown by direct selenization of Pt. Nano Lett. 15, 4013 (2015).
14. Yao, W. et al. Direct observation of spin-layer locking by local Rashba effect in monolayer semiconducting $\mathrm{PtSe}_{2}$ film. Nat. Commun. 8, 14216 (2017).

15. Avsar, A. et al. Defect induced, layer-modulated magnetism in ultrathin metallic $\mathrm{PtSe}_{2}$. Nat. Nanotechnol. 14, 674 (2019).

16. Zhang, W. et al. Magnetism and magnetocrystalline anisotropy in single-layer $\mathrm{PtSe}_{2}$ : interplay between strain and vacancy. J. Appl. Phys. 120, 013904 (2016).

17. Wang, Z., Li, Q., Besenbacher, F. \& Dong, M. Facile synthesis of single crystal PtSe $\mathrm{Pe}_{2}$ nanosheets for nanoscale electronics. Adv. Mater. 28, 10224 (2016).

18. Yan, M. et al. High quality atomically thin $\mathrm{PtSe}_{2}$ films grown by molecular beam epitaxy. 2D Mater. 4, 045015 (2017).

19. Su, T.-Y. et al. Phase-engineered $\mathrm{PtSe}_{2}$-layered films by a plasma-assisted selenization process toward all $\mathrm{PtSe}_{2}$-based field effect transistor to highly sensitive, flexible, and wide-spectrum photoresponse photodetectors. Small 14, 1800032 (2018).

20. Yim, C. et al. High-performance hybrid electronic devices from layered $\mathrm{PtSe}_{2}$ films grown at low temperature. ACS Nano 10, 9550 (2016).

21. Wyckoff, R. W. G. Crystal structures (R. E. Krieger Pub. Co., Malabar, FL, 1964).

22. Croset, B. et al. Measuring surface stress discontinuities in self-organized systems with X rays. Phys. Rev. Lett. 88, 056103 (2002).

23. Martoccia, D. et al. Graphene on $\mathrm{Ru}(0001)$ : a corrugated and chiral structure. New J. Phys. 12, 043028 (2010).

24. Robinson, I. K. \& Tweet, D. J. Surface X-ray diffraction. Rep. Prog. Phys. 55, 599 (1992).

25. Vergnaud, C. et al. Van der Waals solid phase epitaxy to grow large-area manganese-doped $\mathrm{MoSe}_{2}$ few-layers on $\mathrm{SiO}_{2} / \mathrm{Si}$. 2D Mater. 6, 035019 (2019).

26. Cadot, $\mathrm{S}$. et al. Low-temperature and scalable CVD route to $\mathrm{WS}_{2}$ monolayers on $\mathrm{SiO}_{2} / \mathrm{Si}$ substrates. J. Vac. Sci. Technol. A 35, 061502 (2017).

27. Tilinin, I. S., Jablonski, A. \& Werner, W. S. M. Quantitative surface analysis by Auger and x-ray photoelectron spectroscopy. Prog. Surf. Sci. 52, 193 (1996).

28. Nefedov, V. I. \& Fedorova, I. S. Account of photoelectron elastic determination of overlayer thickness, in-depth profiling, escape depth, attenuation coefficients and intensities in surface systems. J. Electron Spectrosc. Rel. Phenomen 85, 221 (1997).

29. Nefedov, V. I. Photoelectron elastic scattering effects in XPS. J. Electron Spectrosc Rel. Phenomen 100, 1 (1999).

30. Band, I. M., Kharitonov, Y. I. \& Trzhaskovskaya, M. B. Photoionization cross sections and photoelectron angular distributions for $\mathrm{X}$-ray line energies in the range 0.132-4.509 keV targets: $1 \leq Z \leq 100$. Atom. Data Nucl. Data Tables 23, 443 (1979).

31. Cantelli, V. The In situ growth of Nanostructures on Surfaces (INS) endstation of the ESRF BM32 beamline: a combined UHV-CVD and MBE reactor for in situ X-ray scattering investigations of growing nanoparticles and semiconductor nanowires. J. Synchrotron. Rad. 22, 688 (2015).

32. Drnec, J. et al. Integration techniques for surface X-ray diffraction data obtained with a two-dimensional detector. J. Appl. Cryst. 47, 365 (2014).

\section{ACKNOWLEDGEMENTS}

The authors acknowledge the financial support from the French ANR project MAGICVALLEY (ANR-18-CE24-0007), the 2DTransformers project under the $\mathrm{OH}$ RISQUE program (ANR-14-OHRI-0004), the French state Equipex funds (ANR-11-EQPX0010, ANR-10-LABX-51-01) (LABEX LANEF) in the framework of the Programme $d^{\prime}$ Investissements d'Avenir. The authors also acknowledge the European Synchrotron Radiation Facility for provision of beam time on the BM32 beamline. Part of this work was performed at the Platform For Nanocharacterization of the CEA Grenoble, MINATEC Campus, with support from the French Recherche Technologique de Base programme. We thank Maurizio De Santis for providing the single crystal substrate used in the experiments.

\section{AUTHOR CONTRIBUTIONS}

G.R. and M.J. conceived and coordinated the project. Discussions in presence of G.R., M.J., O.R., J.C., and R.S. were regularly scheduled to interpret the results and elaborate the next strategies. R.S. and A.M., assisted by T.D. and C.V., prepared the substrate crystals before the allocated beam times; R.S., A.M., S.L., and G.R. performed the experiments during the beam times at $\mathrm{BM} 32$ ( $\mathrm{PtSe}_{2}$ growth and sulfurization, GIXRD characterization); O.R. designed the AR-XPS experiment, and M.G. performed the measurements and the data reduction; R.S., R.H., and X.W. supervised by G.R., quantitatively analyzed the diffraction data; N.G. and O.R. performed the quantitative analysis of the AR-XPS data. R.S., G.R., M.J., J.C., and O.R. cowrote the paper.

\section{COMPETING INTERESTS}

The authors declare no competing interests. 


\section{ADDITIONAL INFORMATION}

Supplementary information is available for this paper at https://doi.org/10.1038/ s41699-020-00175-z.

Correspondence and requests for materials should be addressed to G.R. or M.J.

Reprints and permission information is available at http://www.nature.com/ reprints

Publisher's note Springer Nature remains neutral with regard to jurisdictional claims in published maps and institutional affiliations.
Open Access This article is licensed under a Creative Commons Attribution 4.0 International License, which permits use, sharing, adaptation, distribution and reproduction in any medium or format, as long as you give appropriate credit to the original author(s) and the source, provide a link to the Creative Commons license, and indicate if changes were made. The images or other third party material in this article are included in the article's Creative Commons license, unless indicated otherwise in a credit line to the material. If material is not included in the article's Creative Commons license and your intended use is not permitted by statutory regulation or exceeds the permitted use, you will need to obtain permission directly from the copyright holder. To view a copy of this license, visit http://creativecommons. org/licenses/by/4.0/.

(c) The Author(s) 2020 